\title{
Chiral symmetry breaking in the Wegner-Houghton RG approach
}

\author{
A. Bonanno ${ }^{\mathrm{a}}$ and D. Zappalà ${ }^{\mathrm{b} *}$ \\ ${ }^{a}$ Osservatorio Astrofisico di Catania, Via S. Sofia 58, I-95125, Catania \\ INFN, Sezione di Catania, Corso Italia 57, I- 95129, Catania \\ bINFN, Sezione di Catania and Dip.to di Fisica e Astronomia, Università di Catania, \\ Corso Italia 57, I-95129, Catania, Italy
}

The Wegner-Houghton formulation of the exact renormalization group evolution equation is used in order to study the chiral symmetry breaking of the linear $\sigma$-model coupled to an isospin doublet of quarks. A numerical investigation for a particular truncation of the equation which includes the scalar field renormalization function is presented.

\section{INTRODUCTION}

The full understanding of the phase structure of QCD as the theory of strong interactions has become an important issue since the discovery of asymptotic freedom. The possibility that high temperature QCD could show different properties from the theory at zero temperature has been addressed already in [四]. New features are also predicted for the theory at very high and intermediate baryon density [2], 3, 4,

An essential role is played by the Chiral Symmetry which is supposed to be broken by the vacuum structure of the theory. Thus, before considering the high temperature phase transition with the Chiral Symmetry restoration, one has to deal with the problem of determining a nonzero order parameter which indicates the Chiral Symmetry Breaking (CSB) at zero temperature and density. A strong simplification is obtained by considering the effective theory described by a linear $\sigma$ model of scalar mesons coupled to an isospin multiplet of fermions with the same chiral symmetry group of the original action. Then CSB corresponds to a mexican hat-shaped effective potential and the order parameter is identified with the vacuum expectation value (VEV) of one of the scalar fields.

The infrared (IR) properties of a field theory such as the vacuum structure, can be analysed by means of the wilsonian renormalization group which generates a sequence of effective actions defined at some momentum scale $k$, starting from the original action, by integrating out in the latter all the modes with frequency higher than $k$. This leads to the construction of a differential flow equation for the $k$-dependent action, generally known as Exact Renormalization Group Equation (ERGE). In the past years many analytical and numerical approaches to the ERGE, applied to various theories, have been developed. Two recent extensive reviews of this topic are [5,6] and a detailed ERGE analysis of the Chiral Phase Transition can be found in [7 [10].

\footnotetext{
*Talk given by D. Zappalà
} 
Here we shall consider just the simple case of the CSB at zero temperature employing the sharp cut-off version of the ERGE, namely the Wegner-Hougton equation [11]. The cutoff dependence of the ERGE for the $\mathrm{O}(4)$ linear $\sigma$-model has been addressed in [12 where, however only the flow of the scalar potential is considered while the Yukawa coupling is kept fixed. In addition to the Yukawa coupling flow, we are particularly interested in the behavior of the field renormalization. In fact, if our model is really an effective theory which, at some scale, can naturally replace the original QCD action, then at this scale one expects the formation of the mesonic bound states characterized by a very small wave function renormalization function. The reduction of QCD to the quark-meson action has been analysed before and a review of it is given in [10]. As expected the value of the scalar field renormalization is vanishingly small at the mesonic bound state formation scale which is found between 0.60 and $0.63 \mathrm{GeV}$. Then, the flow of the field renormalization deserves particular attention.

\section{ANALYSIS AND RESULTS}

The explicit form of the euclidean action at a scale $k$ is $\left(\rho \equiv \sigma^{2}+\vec{\pi}^{2}\right)$

$$
S_{k}=\int d^{4} x\left(\frac{z_{l k}}{2} \partial_{\mu} \sigma \partial_{\mu} \sigma+\frac{z_{t k}}{2} \partial_{\mu} \vec{\pi} \partial_{\mu} \vec{\pi}+U_{k}(\rho)+\sum_{c}^{N_{c}} \bar{\psi}_{c}\left(i \gamma \cdot \partial+g_{k} \sigma+i g_{k} \gamma^{5} \vec{\pi} \vec{\tau}\right) \psi_{c}\right)(1)
$$

$\psi_{c}$ is a flavor doublet and the summation over the index $c$ is extended to $N_{c}$ colors. No CSB fermion mass term is included and the action is fully invariant under chiral transformations. The presence of CSB depends on the shape of the $\mathrm{O}(4)$ symmetric potential $U_{k}$ in the limit $k \rightarrow 0 . z_{l k}$ and $z_{t k}$ are the $\sigma$ and $\pi$ renormalization functions. As long as $U_{k}$ has zero vacuum expectation value (VEV), we choose the following parametrization $U_{k}=(1 / 2) m_{k}^{2} \rho+\left(\lambda_{k} / 24\right) \rho^{2}$ and consider the flow of the two parameters $m_{k}^{2}$ and $\lambda_{k}$ neglecting all the irrelevant operators that could be generated during the flow. In the presence of a nonvanishing $\mathrm{VEV} \bar{\rho}_{k}$, according to [12], we reparametrize the potential in terms of $\bar{\rho}_{k}$ and $\lambda_{k}$, recalling that $m_{k}^{2}$ can be expressed in term of these two parameters through the minimum condition. The purpose of this double choice is practical, we take the couple of parameters which corresponds to the simplest set of flow equations.

The Wegner-Houghton equation can be reduced to a set of coupled ordinary differential equations for the $k$ dependent parameters in (1), i.e. $g_{k}, z_{l k}, z_{t k}, \lambda_{k}$ and $m_{k}^{2}$ or $\bar{\rho}_{k}$. In particular the equations for the parameters in the potential are deduced in [12], and the one for $g_{k}$ can be obtained by an analogous procedure. The equations for $z_{l k}, z_{t k}$, (which coincide as long as $\bar{\rho}_{k}=0$, according to the chiral simmetry) are deduced following the procedure formulated in [13] and already implemented in [14]. In the following, the derivatives of $z_{l k}$ and $z_{t k}$ w.r.t. the fields, which are supposed to be small, are neglected.

The pion decay constant and the constituent fermion mass are two IR constraints for our equations. In fact, in the limit $k \rightarrow 0$ we have $\sqrt{z_{l k} \bar{\rho}_{k}} \equiv f \rightarrow f_{\pi} \sim 0.09 \mathrm{Gev}$ and $g_{k} \sqrt{\bar{\rho}_{k}} \equiv M \rightarrow M_{q} \sim 0.3 \mathrm{GeV}$, as initial conditions for the equations. If we start, according to [10, the flow at the UV scale $k=0.6 \mathrm{GeV}$ with a convex potential $\left(m_{k}^{2}>0\right)$, then at a certain scale $K_{\chi}, m_{k}^{2}$ becomes negative generating a nonzero $\bar{\rho}_{k}$. In the following the three remaining initial conditions imposed are $\bar{\rho}_{k}=0$ at the CSB scale $K_{\chi}$ and a particular value for $z_{l k}$ at the UV scale (the symmetry requires $z_{l k}=z_{t k}$ at the UV scale). 


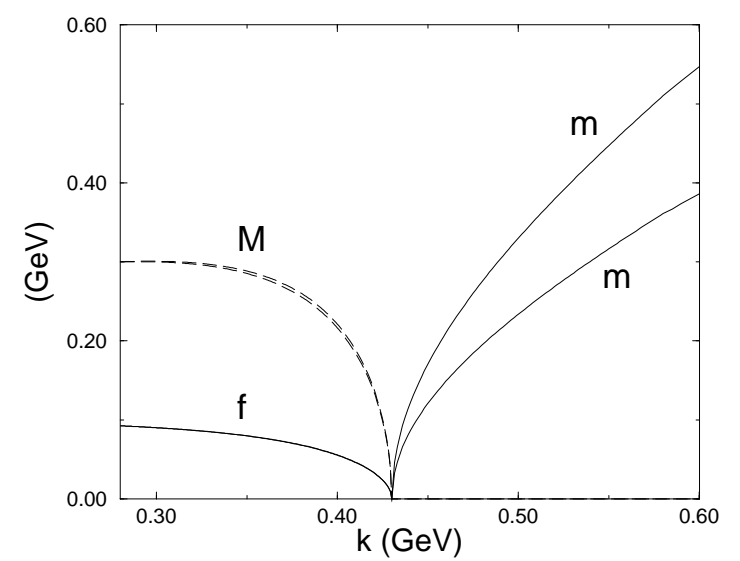

Figure 1. RG flow with two different sets of initial conditions (see text). For these two sets, the quark mass $M$ and the decay constant $f$ are displayed below $K_{\chi}=$ $0.43 \mathrm{GeV}$, and the scalar mass $m$ above $K_{\chi}$.

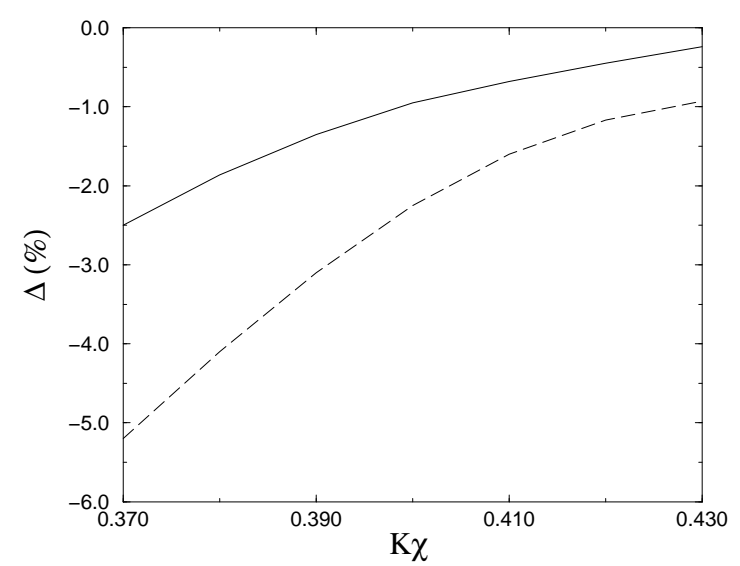

Figure 2. $100 \times \Delta$ (see text) vs. $K_{\chi}$ $(\mathrm{GeV})$ for two different values of $M_{q} . M_{q}=$ $0.30 \mathrm{GeV}$ (solid) and $M_{q}=0.33 \mathrm{GeV}$ (dashed).

Figure 1 shows, above $K_{\chi}$ (fixed at the value $K_{\chi}=0.43 \mathrm{GeV}$ ), the flow of the scalar mass $m$ (the subscript $k$ is omitted) for two different initial values of $z_{l}\left(=z_{t}\right)$ and, below $K_{\chi}$, the corresponding $f$ and $M$. The curves for $f$ and $M$ in the two cases are almost superposed indicating insensitivity to the UV condition on $z_{l}$. The value of $K_{\chi}$ is suggested by an IR stability criterion. In order to use a particular value of quark mass, $M_{q}$, as the initial condition for the running quantity $M$ defined above, $M$ must be practically flat around $k=M_{q}$. The same criterion should apply to $f_{\pi}$ but, since $f_{\pi}$ is smaller than $M_{q}$, we find that $f$ at the scale $k=f_{\pi}$ is already a stable parameter. After fixing the IR condition $M\left(k=M_{q}-\epsilon\right)=M_{q}$ with $\epsilon=0.03 \mathrm{GeV}$, we have considered the ratio $\Delta=\left(M\left(k=M_{q}\right)-M_{q}\right) / M_{q}$, plotted vs. $K_{\chi}$ in Figure 2 for two different values of $M_{q}$. The stability requirement indicates that $K_{\chi}$ should not be smaller than $\sim 0.4 \mathrm{Gev}$.

In Figures 3 and t the other running parameters are displayed for the same two initial values of $z_{l}$ that have been used in Figure 1. It should be noted the small running of $g$ and $\lambda$, in the range of $k$ considered, and their large values which are clearly nonperturbative. As shown in Figure 4 , when going from the UV to the IR scale, the field renormalizations grow and, below $K_{\chi}, z_{l}$ and $z_{t}$ increase differently. Since reasonably we expect, for the pion physics at scales around $k=0.3 \mathrm{GeV}$, field renormalization functions close to the unity, we must require very small values at $k=0.6 \mathrm{GeV}$. Namely we used $z_{l}=z_{t}=0.1$ and $z_{l}=z_{t}=0.01$ in the two cases here considered in Figures 1, 1, 3, 田. Higher curves for $m, \lambda, g$ correspond to the former case, lower curves to the latter.

The approximation here employed for the ERGE has been sufficient for exploring a nonperturbative region (note that, as explained in [10], the renormalized couplings which include the field renormalization effect, are larger than those in Figure (3) and it has 


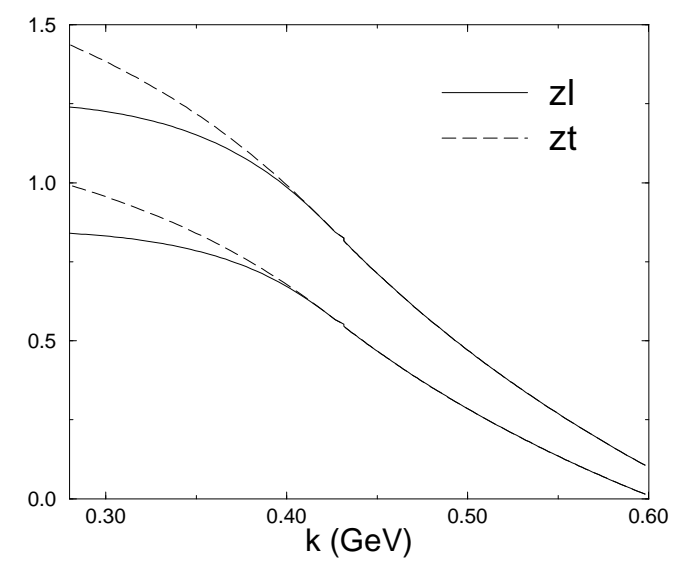

Figure 3. Flow of $z_{l}$ (solid) and $z_{t}$ (dashed) for two different sets of initial data (see text). $z_{l}$ and $z_{t}$ coincide above $K_{\chi}=0.43$.

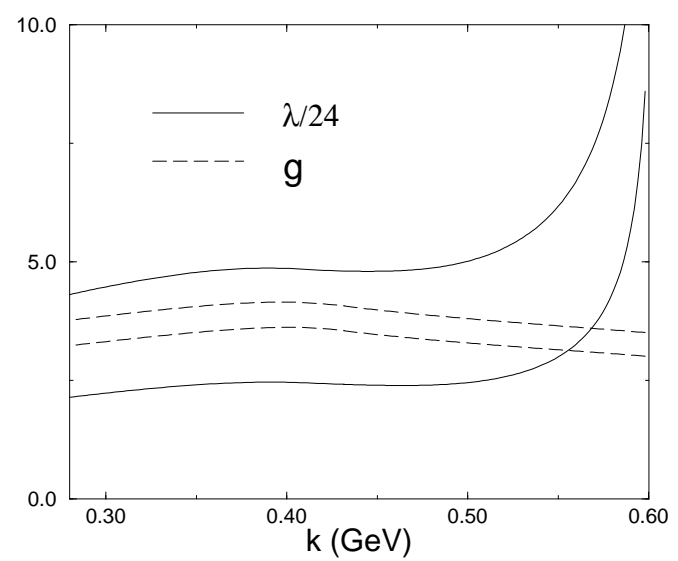

Figure 4. Flow of the scaled scalar quartic coupling $\lambda / 24$ (solid) and of the Yukawa coupling $g$ (dashed) for two different sets of initial data (see text).

yielded a very small (vanishing) scalar field renormalization around $0.6 \mathrm{GeV}$. We hope to extend our analysis to the finite temperature case.

\section{REFERENCES}

1. J.C. Collins and M.J. Perry, Phys. Rev. Lett. 34 (1975) 1353.

2. D. Bailin and A. Love, Phys. Rep. 107 (1984) 325.

3. M. Alford, K. Rajagopal and F. Wilczek, Phys. Lett. 422B (1998) 247.

4. R. Rapp, T. Shafer, E.V. Shuryak and M. Velkovsky, Phys. Rev. Lett. 81 (1998) 53.

5. C. Bagnuls and C. Bervillier, Exact renormalization group equations. An introductory review, Preprint e-Print Arch.:hep-th/0002034, Feb 2000.

6. J. Berges, N. Tetradis, C. Wetterich, Nonperturbative renormalization flow in quantum field theory and statistical physics, Preprint MIT-CTP-2980, HD-THEP-00-26, e-Print Arch.:hep-ph/0005122, May 2000. Submitted to Phys. Rep.

7. J. Berges, N. Tetradis and C. Wetterich, Phys. Rev. Lett. 77 (1996) 873.

8. D.-U. Jungnickel and C. Wetterich, Phys. Rev. D53 (1996) 5142.

9. J. Berges, D.-U. Jungnickel and C. Wetterich, Phys. Rev. D59 (1999) 034010.

10. J. Berges, QCD in extreme conditions and the wilsonian exact renormalization group, Preprint MIT-CTP-2829, e-Print Arch.: hep-ph/9902419.

11. F.J. Wegner and A. Houghton, Phys. Rev. A8 (1972) 401.

12. G. Papp, B.J. Schaefer, H.J. Pirner and J. Wambach, Phys. Rev. D61 (2000) 096002.

13. C. Fraser, Z. Phys. C28 (1985) 101.

14. A. Bonanno and D. Zappalà, Phys. Rev. D56 (1997) 3759. 